\title{
Author Correction: Mitochondrial UPR through generations
}

Mooncheol Park and Meng C. Wang (10)

Correction to: Nature Cell Biology https://doi.org/10.1038/s41556-021-00729-3, published online 2 August 2021.

In the version of this News \& Views initially published, in the first paragraph, fifth sentence, in the text reading "which is mediated by the Piwi/PRG-1 argonaute-dependent piRNA (Piwi-interacting RNA) pathway and the TGF- $\beta$ ligand DAF-17...", the term "DAF-17" has been corrected to read "DAF-7."

This change has been made in the online version of the News $\&$ Views.

Published online: 16 September 2021

https://doi.org/10.1038/s41556-021-00757-z

(C) Springer Nature Limited 2021 